\title{
Bangpungtongseong-san, a traditional herbal medicine, attenuates chronic asthmatic effects induced by repeated ovalbumin challenge
}

\author{
MEE-YOUNG LEE, IN-SIK SHIN, WOO-YOUNG JEON, NARA SHIN and HYEUN-KYOO SHIN
}

Basic Herbal Medicine Research Group, Korea Institute of Oriental Medicine, Daejeon 305-811, Republic of Korea

Received October 2, 2013; Accepted February 5, 2014

DOI: $10.3892 /$ ijmm.2014.1654

\begin{abstract}
Airway remodeling is characterized by airway wall thickening, subepithelial fibrosis, increased smooth muscle mass, angiogenesis and increased mucus secretion, which can lead to chronic and obstinate asthma and can obstruct pulmonary function. In this stuyd, the effects of Bangpungtongseong-san water extract (BPTS) on airway remodeling were examined using a murine model of bronchial asthma induced by ovalbumin (OVA) challenge. We focused on the effects of BPTS on the regulation of chronic asthma. BALB/c mice were randomly assigned to 5 groups, some of which were sensitized and challenged with OVA for 4 weeks. After the final ovalbumin challenge, typical asthma-like morphological changes were observed in the lung tissue with hematoxylin and eosin staining, periodic acid-Schiff, as well as with Masson's trichrome staining. The levels of transforming growth factor- $\beta 1$ (TGF- $\beta 1$ ) and Smad3 were assessed by immunohistochemistry and western blot analysis. The expression levels of vascular endothelial growth factor (VEGF) and adhesion molecules, such as intercellular adhesion molecule-1 and vascular cell adhesion molecule-1 were also detected by western blot analysis. Our results revealed that BPTS reduced the OVA-induced increase in the infiltration of leukocytes, mucus hyperplasia and collagen deposition. Compared with the OVA-challenged group, the BPTS group had lower expression levels of adhesion molecules, TGF- $\beta 1$, Smad3 and VEGF proteins in the lung tissues. The results of the current study suggest that BPTS prevents asthma airway remodeling in chronic asthma by inhibiting the activation of the TGF- $\beta 1$-Smad3-signaling pathway, as well as the expression of VEGF and adhesion molecules. BPTS may thus be a potential drug for the treatment of patients with changes that occur in the airways due to severe asthma.
\end{abstract}

Correspondence to: Dr Hyeun-Kyoo Shin, Basic Herbal Medicine Research Group, Korea Institute of Oriental Medicine, 483 Expo-ro, Yusung-gu, Daejeon 305-811, Republic of Korea

E-mail: cozy37@gmail.com; hkshin@kiom.re.kr

Key words: chronic asthma, transforming growth factor- $\beta 1$, Smad3, vascular endothelial growth factor, adhesion molecule

\section{Introduction}

Chronic airway disorders, including chronic obstructive pulmonary disease, cystic fibrosis and asthma, are associated with persistent pulmonary inflammation, airway remodeling, goblet cell metaplasia and collagen deposition (1), which significantly contribute to morbidity and mortality worldwide. The incidence and severity of chronic lung diseases are constantly increasing. Chronic lung diseases affect from 100 to 150 million individuals worldwide and are associated with a significantly high mortality rate (2). The pathogenesis of asthma was originally thought to be a T helper 2 (Th2)-mediated disease, although proinflammatory cytokines also play significant and distinct roles in the pathogenesis of asthma. Th2 cytokines, such as interleukin (IL)-4, IL-5, IL-9 and IL-13 induce changes in the airways and lung parenchyma, which in turn lead to airway eosinophilia, epithelial cell proliferation with goblet cell hyperplasia, increased mucus secretion, smooth muscle hyperplasia, subepithelial fibrosis, immunoglobulin E (IgE) secretion and an increased production of chemokines that attract $\mathrm{T}$ cells, eosinophils, neutrophils and mast cells (3). Th2 cytokines, such as IL-5-activate eosinophils $(4,5)$, whose migration in turn induces eosinophilic airway inflammation. IL-4 directly promotes the characteristics of asthma, such as Ig isotype switching in B cells to primarily produce IgE and IgG1 $(6,7)$. IL-13 production has been observed in the skin and gingival fibroblasts $(8,9)$. Transforming growth factor $\beta 1$ (TGF- $\beta 1$ ) and IL-13 activate lung fibroblasts in patients with asthma (10). In the asthmatic patient, eosinophils produce profibrotic and proangiogenic cytokines, such as TGF- $\beta 1$ and vascular endothelial growth factor (VEGF) (11). TGF- $\beta 1$ expression is increased in patients with asthma, and this increase seems to correlate with disease severity and the degree of peribronchial fibrosis (12).

VEGF has been shown to play a critical role in increasing Th2 cell-mediated inflammation and TGF- $\beta 1$ production (13), which is consistent with the finding that the inhibition of VEGF expression attenuates peribronchial fibrosis by blocking TGF- $\beta 1$ production (14). The recruitment and lung infiltration of eosinophils are controlled by chemokines and adhesion molecules. Vascular cell adhesion molecule 1 (VCAM-1) is expressed on activated endothelial cells and contributes to the firm adhesion of eosinophils to vascular endothelial cells (15). Intercellular adhesion molecule 1 (ICAM-1) is an endothelial transmembrane protein that facilitates the endothelial 
Table I. Crude components contained in Bangpungtongseong-san (BPTS) extract.

\begin{tabular}{lccc}
\hline Scientific name & Amount (g) & Company of purchase & Source \\
\hline Talcum & 6.375 & HMAX & China \\
Glycyrrhiza uralensis & 4.5 & HMAX & China \\
Gypsum & 2.625 & HMAX & China \\
Scutellaria baicalensis & 2.625 & HMAX & Jeongseon, Korea \\
Platycodon grandiflorum & 2.625 & Omniherb & Yeongcheon, Korea \\
Ledebouriella seseloides & 1.6875 & HMAX & China \\
Cnidium officinale & 1.6875 & Omniherb & Yeongcheon, Korea \\
Angelica gigas & 1.6875 & Omniherb & Pyeongchang, Korea \\
Paeonia lactiflora & 1.6875 & Omniherb & Hwasun, Korea \\
Rheum undulatum & 1.6875 & HMAX & China \\
Ephedra sinica & 1.6875 & HMAX & China \\
Mentha pulegium & 1.6875 & Omniherb & China \\
Forsythia koreana & 1.6875 & HMAX & China \\
Erigeron canadensis & 1.6875 & HMAX & China \\
Schizonepeta tenuifolia & 1.3125 & Omniherb & China \\
Atractylodes japonica & 1.31 & HMAX & China \\
Gardenia jasminoides & 1.3 & Omniherb & Muju, Korea \\
Zingiber officinale & 6.25 & Omniherb & Yeongcheon, Korea \\
Total & 44.125 & & \\
\hline
\end{tabular}

transmigration of eosinophils into different pathological environments (16).

Bangpungtongseong-san (BPTS) is a major traditional herbal medicine widely used in the treatment of obesity (17). BPTS has been reported to inhibit atherosclerosis (18), obesity (19), hypertension (20) and allergic rhinitis (21). However, to the best of our knowledge, the effects of BPTS on airway inflammation and lung fibrosis in chronic asthma have not yet been described. Glucocorticoids are anti-inflammatory medications and are often used as maintenance therapy in patients with acute and chronic asthma, despite the fact that some of them are steroid resistant. There is a great need for a more effective treatment for chronic asthma, with fewer undesired side-effects. In the present study, we examined whether BPTS exerts inhibitory effects on airway inflammation and pulmonary fibrosis in a mouse model of chronic asthma induced by repeated ovalbumin (OVA) challenge.

\section{Materials and methods}

Preparation of BPTS extract. BPTS was prepared in our laboratory from a mixture of chopped crude herbs purchased from Omniherb (Yeongcheon, Korea) and HMAX (www.HMAX. co.kr; Chungbuk, Korea). BPTS was prepared from a mixture of herbs (shown in Table I) and was extracted in distilled water at $100^{\circ} \mathrm{C}$ for $2 \mathrm{~h}$. The extract was evaporated to dryness and freeze-dried (yield, 22.21\%). The composition of BPTS was analyzed using high-performance liquid chromatography (HPLC) as previously described (22). The chemical standards used to identify and quantify the compounds in the BPTS were geniposide, liquiritin, baicalin and glycyrrhizin.
Animals and environmental conditions. Specific pathogenfree female BALB/c mice (7 weeks old) were purchased from Orient Co. (Seoul, Korea) and maintained in an animal facility under standard laboratory conditions for 1 week before the experiments were conducted. The animals were provided with water and standard chow ad libitum. All the experimental procedures were carried out in accordance with the National Institutes of Health (NIH) Guidelines for the Care and Use of Laboratory Animals and were approved by the Animal Care and Use Committee of Chungnam National University, Daejeon, Korea. The animals were cared for in accordance with the National Animal Welfare Law of Korea.

Experimental protocol. Specific pathogen-free female BALB/c mice $(n=35)$ were sensitized on days 0 and 14 by an intraperitoneal injection of $20 \mu \mathrm{g}$ of OVA, emulsified with $2 \mathrm{mg}$ of aluminum hydroxide in $200 \mu \mathrm{l}$ of phosphate-buffered saline (PBS) (pH 7.4). On day 21, the mice received an airway challenge with OVA [1\% (w/v)] for $1 \mathrm{~h}$ with the use of an ultrasonic nebulizer (NE-U12; Omron Corp., Tokyo, Japan); this step was repeated 3 times per week, for 4 weeks. Each day during the 4 weeks of the airway challenge, BPTS was freshly prepared in PBS and administered by gavage at $50 \mathrm{mg} / \mathrm{kg}$ or $100 \mathrm{mg} / \mathrm{kg}$ of body weight. The mice were divided into 5 groups as follows ( $\mathrm{n}=7$ per group): $\mathrm{PBS} / \mathrm{PBS}$, normal control mice treated with PBS only; OVA/PBS; OVA-sensitized/challenged mice treated orally with PBS; OVA/montelukast (Mon), OVA-sensitized/ challenged mice treated orally with montelukast $(30 \mathrm{mg} / \mathrm{kg})$; OVA/BPTS-50, OVA-sensitized/challenged mice treated orally with BPTS $(50 \mathrm{mg} / \mathrm{kg})$; and OVA/BPTS-100, OVA-sensitized/ challenged mice treated orally with BPTS $(100 \mathrm{mg} / \mathrm{kg})$. 


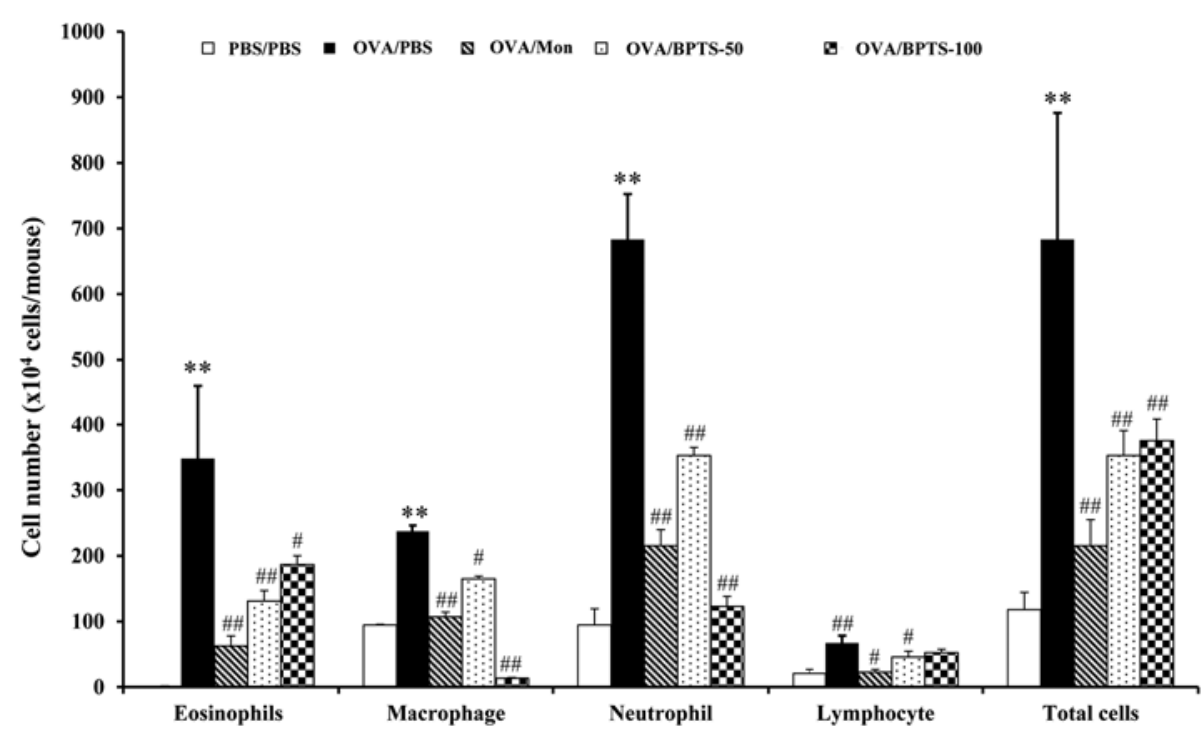

Figure 1. Bangpungtongseong-san water extract (BPTS) inhibits the recruitment of inflammatory cells in bronchoalveolar lavage fluid (BALF) of mice $48 \mathrm{~h}$ after the final ovalbumin (OVA) challenge. Cells were isolated by centrifugation and stained with Diff-Quik stain reagent. With the use of light microscopy after having counted cells in at least 5 squares of a hemocytometer and having excluded dead cells with the use of Trypan blue, all inflammatory cells were calculated. Phosphate-buffered saline (PBS)/PBS, normal control mice treated with PBS only; OVA/PBS, OVA-sensitized/challenged mice treated orally with PBS; OVA/montelukast (Mon), OVA-sensitized/challenged mice montelukast $(30 \mathrm{mg} / \mathrm{kg}) ;$ OVA/BPTS-50, OVA-sensitized/challenged mice treated orally with BPTS $(50 \mathrm{mg} / \mathrm{kg}) ;$ OVA/BPTS-100, OVA-sensitized/challenged mice treated orally with BPTS $(100 \mathrm{mg} / \mathrm{kg})$. Values are expressed as the means \pm SEM $\left(\mathrm{n}=7 /\right.$ group). ${ }^{* *}$ Significantly different from PBS/PBS, $\mathrm{P}<0.01$; $* \#, * \|$ significantly different from $\mathrm{OVA} / \mathrm{PBS}, \mathrm{P}<0.05$ and $<0.01$, respectively.

At the end of the 4 weeks of OVA challenge, bronchoalveolar lavage fluid (BALF) samples were obtained for analysis, as previously described (22). In brief, the mice were sacrificed $48 \mathrm{~h}$ after the final challenge by an intraperitoneal injection of pentobarbital $(50 \mathrm{mg} / \mathrm{kg}$; Hanlim Pharmaceutical Co., Seoul, Korea), and a tracheostomy was performed. To obtain BALF, ice-cold PBS $(0.5 \mathrm{ml})$ was infused into the lungs 3 times and withdrawn each time via tracheal cannulation (total volume $1.5 \mathrm{ml}$ ). Total inflammatory cell numbers were determined by counting cells in at least 5 squares of a hemocytometer, after having excluded the dead cells by Trypan blue staining. To determine the differential cell count, $100 \mu \mathrm{l}$ of BALF were centrifuged $\left(200 \mathrm{x} \mathrm{g}, 4^{\circ} \mathrm{C}, 10 \mathrm{~min}\right)$ onto slides using a Cytospin unit (Hanil Science Industrial, Seoul, Korea). The slides were dried, and the cells were fixed and stained using Diff-Quik ${ }^{\circledR}$ staining reagent (B4132-1 A; IMEB Inc., San Marcos, CA, USA) according to the manufacturer's instructions. The supernatant obtained from the BALF Cytospin was stored at $-70^{\circ} \mathrm{C}$ for biochemical analysis.

Determination of total cell, eosinophil, lymphocyte, neutrophil and macrophage cell counts in BALF. Differential cell counting was performed as previously described (22).

Measurement of cytokine and chemokine levels in BALF. The levels of IL-4, IL-13, IL-33, tumor necrosis factor- $\alpha$ (TNF- $\alpha$ ) and eotaxin in BALF were measured using enzyme-linked immunosorbent assay (ELISA) kits according to the manufacturer's instructions (BioSource International, Camarillo, CA, USA) as previously described (22).

Measurement of TGF- $\beta 1$ and VEGF expression levels in lung tissue. $\mathrm{c}$ and VEGF expression levels in BALF were measured using the respective ELISA kits according to the manufacturers' instructions (TGF- $\beta 1$, R\&D Systems, Inc., Minneapolis, MN, USA; and VEGF, Immuno-Biological Laboratories Co., Ltd., Minneapolis, MN, USA). Total protein concentration was measured with a kit assay (Bio-Rad, Hercules, CA, USA). The results are expressed as $\mathrm{pg} / \mathrm{mg}$ protein.

Measurement of total and OVA-specific IgE expression levels in BALF and plasma. Serum was collected via centrifugation ( $200 \mathrm{x} \mathrm{g}, 10 \mathrm{~min}$ ) and stored at $-70^{\circ} \mathrm{C}$. Total and OVA-specific $\mathrm{IgE}$ levels were measured using ELISA as previously described (22).

Immunoblot analysis. Equal amounts of total lung protein $(30 \mu \mathrm{g})$ were heated at $100^{\circ} \mathrm{C}$ for $5 \mathrm{~min}$, loaded onto $8 \%$ sodium dodecyl sulfate polyacrylamide gels and electrophoresed. The proteins were then transferred onto nitrocellulose membranes (at $100 \mathrm{~V}$ for $2 \mathrm{~h}$ ), and the membranes were blocked for $1 \mathrm{~h}$ with Tris-buffered saline containing $0.05 \%$ Tween-20 (TBST) plus 5\% skim milk. The blocked membranes were incubated with primary antibodies against VEGF, VCAM-1, ICAM-1, TGF- $\beta 1$ and Smad3 (all at a 1:1,000 dilution; Abcam, Cambridge, MA, USA) and anti- $\beta$-actin antibody (1:1,000 dilution; Cell Signaling Technology, Inc., Danvers, MA, USA) overnight at $4^{\circ} \mathrm{C}$. The membranes were washed three times with TBST and then incubated with a horseradish peroxidase-conjugated secondary antibody (1:3,000 dilution; Jackson ImmunoResearch Laboratories, Inc., West Grove, PA, USA) for $1 \mathrm{~h}$ at room temperature. The membranes were washed another 3 times with TBST and developed using the enhanced chemiluminescence kit (Amersham Pharmacia Biotech, Uppsala, Sweden) according to the manufacturer's instructions. For quantitative analysis, densitometric band values were determined using ChemiDoc (Bio-Rad). 

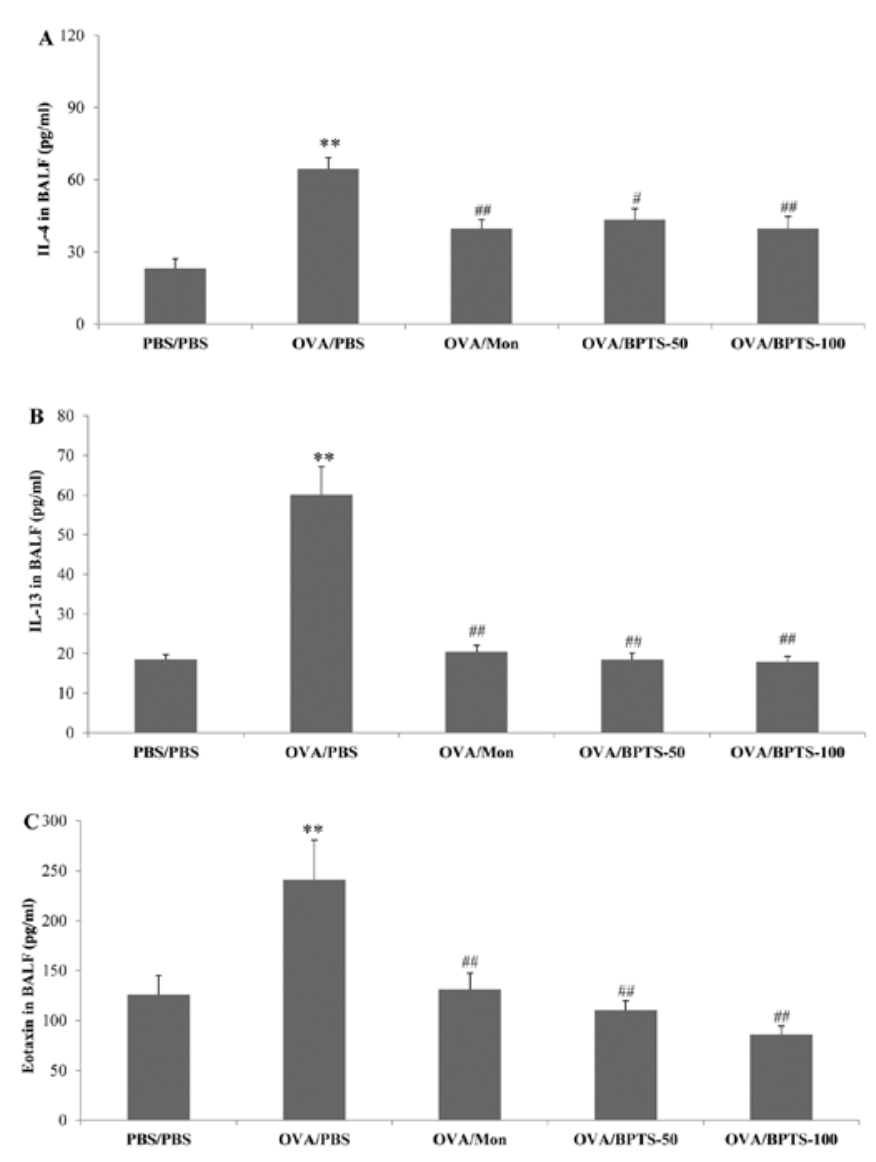

Figure 2. Bangpungtongseong-san water extract (BPTS) reduces the levels of (A) IL-4, (B) IL-13, and (C) eotaxin in bronchoalveolar lavage fluid (BALF) of mice $48 \mathrm{~h}$ after the final OVA challenge. Phosphate-buffered saline (PBS)/PBS, normal control mice treated with PBS only; OVA/PBS, OVAsensitized/challenged mice treated orally with PBS; OVA/montelukast (Mon), OVA-sensitized/challenged mice treated orally with montelukast $(30 \mathrm{mg} / \mathrm{kg})$; OVA/BPTS-50, OVA-sensitized/challenged mice treated orally with BPTS (50 mg/kg); OVA/BPTS-100, OVA-sensitized/challenged mice treated orally with BPTS $(100 \mathrm{mg} / \mathrm{kg})$. Values are expressed as the means $\pm \mathrm{SEM}$

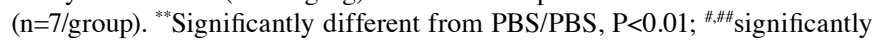
different from OVA/PBS, $\mathrm{P}<0.05$ and $<0.01$, respectively

Histological analysis. For histological examination, before the lungs were removed, the left lungs were filled intratracheally with a fixative $(0.8 \%$ formalin, $4 \%$ acetic acid) using a ligature around the trachea. Tissues were embedded in paraffin, sectioned at $4 \mu \mathrm{m}$ thickness, and stained with hematoxylin and eosin (H\&E) solution (MHS-16 and HT110-1-32 respectively; Sigma, St. Louis, MO, USA) and periodic acid-Schiff (PAS) (IMEB Inc.) in order to estimate inflammation and mucus production, respectively. To evaluate peribronchial fibrosis, the sections were stained with Masson's trichrome.

For immunohistochemistry, the paraffin sections were deparaffinized, dehydrated, washed in PBS with $0.3 \%$ Triton X-100 and pre-incubated for $10 \mathrm{~min}$ at room temperature with $10 \%$ goat serum to block non-specific staining. The slides were then incubated with primary mouse TGF- $\beta 1$ antibodies (1:100 dilution, Abcam) overnight at $4^{\circ} \mathrm{C}$. The following day, the primary antibodies were removed, the sections were washed and incubated with biotinylated secondary antibody at $37^{\circ} \mathrm{C}$ for $1 \mathrm{~h}$, and then incubated with an avidin-biotin-peroxidase complex (Vector Laboratories Inc., Burlingame, CA, USA) at room
Table II. Levels of total IgE and ovalbumin (OVA)-specific $\mathrm{IgE}$ in bronchoalveolar lavage fluid (BALF) and plasma.

\begin{tabular}{lcc}
\hline & \multicolumn{1}{c}{ BALF } & Plasma \\
\cline { 2 - 3 } Group & $\begin{array}{c}\text { Total IgE } \\
(\mathrm{ng} / \mathrm{ml})\end{array}$ & $\begin{array}{c}\text { OVA-specific IgE } \\
(\mathrm{ng} / \mathrm{ml})\end{array}$ \\
\hline PBS/PBS & $1.50 \pm 1.11$ & $<0$ \\
OVA/PBS & $32.55 \pm 17.94^{\mathrm{a}}$ & $204.91 \pm 37.72^{\mathrm{a}}$ \\
OVA/Mon & $14.42 \pm 4.30^{\mathrm{c}}$ & $101.30 \pm 46.93^{\mathrm{c}}$ \\
OVA/BPTS-50 & $15.49 \pm 4.99^{\mathrm{c}}$ & $115.21 \pm 44.78^{\mathrm{c}}$ \\
OVA/BPTS-100 & $19.65 \pm 5.87^{\mathrm{b}}$ & $185.22 \pm 22.99$ \\
\hline
\end{tabular}

PBS/PBS, normal control mice treated with PBS only; OVA/PBS, OVA-sensitized/challenged mice treated orally with PBS; OVA/Mon, OVA-sensitized/challenged mice treated orally with montelukast (30 mg/kg); OVA/BPTS-50, OVA-sensitized/challenged mice treated orally with BPTS $(50 \mathrm{mg} / \mathrm{kg}$ ); OVA/BPTS-100; OVA-sensitized/ challenged mice treated orally with BPTS $(100 \mathrm{mg} / \mathrm{kg}) .{ }^{\mathrm{a}} \mathrm{P}<0.01$, significantly different from the PBS/PBS group; ${ }^{\text {b,c }} \mathrm{P}<0.05$ and $<0.01$, significantly different from the OVA/PBS group, respectively.

temperature for $1 \mathrm{~h}$. After the excess complex was removed, the sections were washed with PBS and incubated with $0.05 \%$ diaminobenzidine (1:200; Millipore, Billerica, MA, USA) for a further $10 \mathrm{~min}$. The sections were counterstained, rinsed in PBS to terminate the reaction, and protected with cover slips for microscopic examination.

Statistical analysis. Data are presented as the means \pm SD. Statistical significance was determined using analysis of variance followed by a multiple comparison test with Bonferroni adjustment. Values of $\mathrm{p}<0.05$ or $<0.01$ were considered to indicate statistically significant differences.

\section{Results}

$B P T S$ reduces the number of inflammatory cells in BALF. The effects of BPTS were investigated on various cell types present in BALF. The repeated challenge with OVA causes infiltration predominantly by neutrophils. As shown in Fig. 1, the number of neutrophils, lymphocytes, macrophages, eosinophils and total cells in BALF 2 decreased significantly in a dose-dependent manner following treatment with BPTS.

BPTS decreases the levels of $I L-4, I L-13$ and eotaxin in $B A L F$. The levels of IL-4, IL-13 and eotaxin in BALF were significantly higher in the OVA-sensitized/challenged mice (OVA/PBS) compared with the PBS/PBS mice (Fig. 2). The BPTS-treated mice presented significantly lower levels of these 3 compounds compared with the OVA/PBS mice. Similar to the Th2-type cytokine levels, the eotaxin levels increased in the OVA/PBS group and decreased in a dosedependent manner in the BPTS-treated group (Fig. 2C).

BPTS decreases the expression levels of total IgE and $O V A$-specific IgE in BALF and plasma. The expression levels 

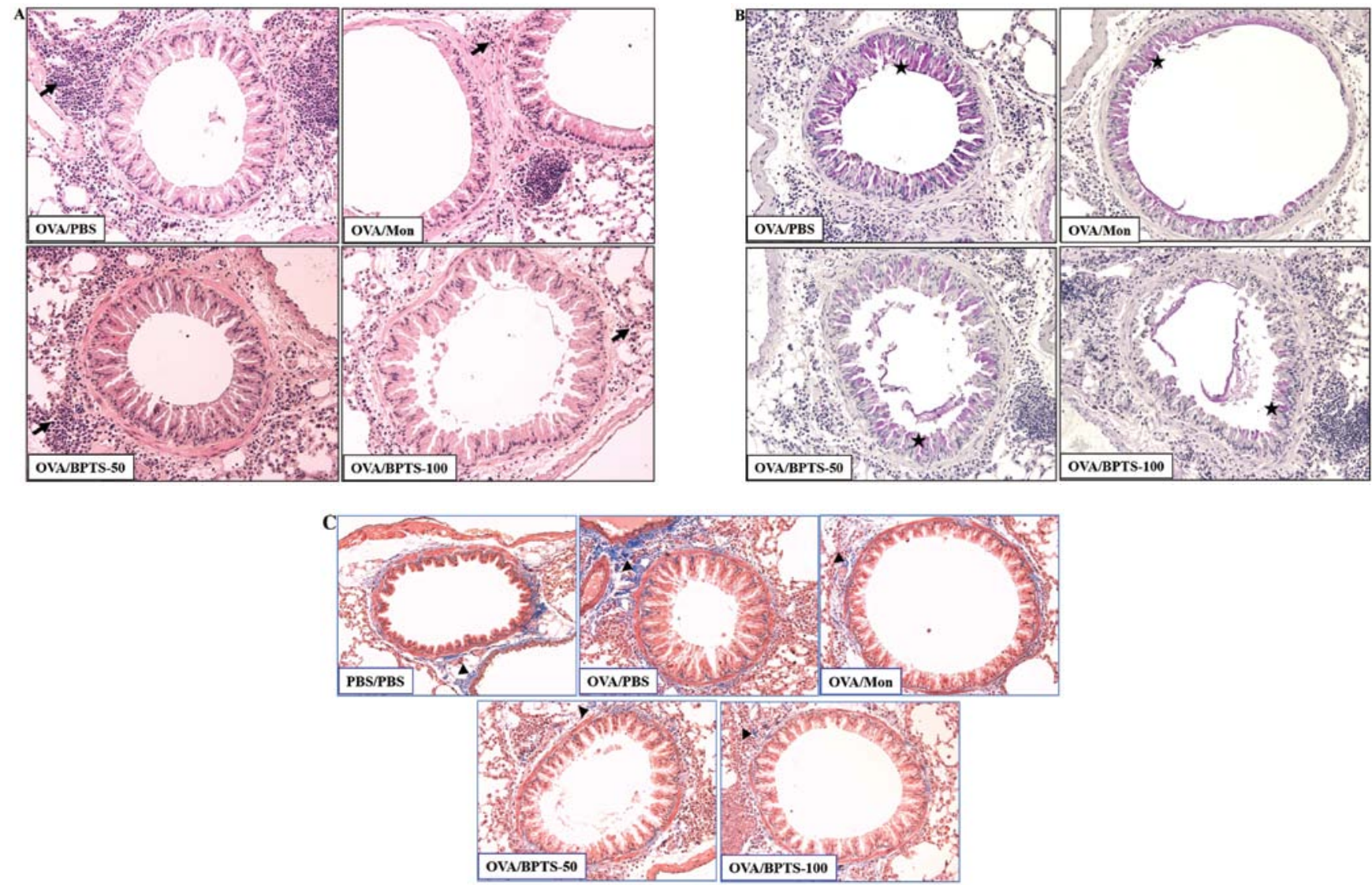

D

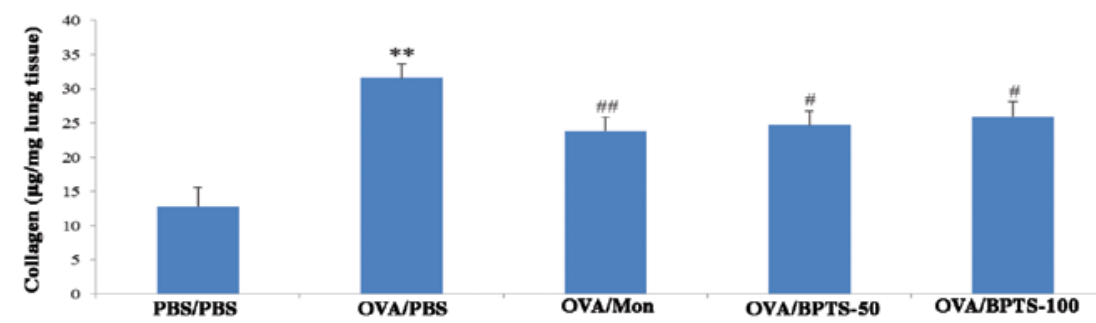

Figure 3. Bangpungtongseong-san water extract (BPTS) attenuates airway inflammation (arrows), mucus production (asterisks), and fibrosis (arrowheads) in lung tissue $48 \mathrm{~h}$ after the final ovalbumin (OVA) challenge. Histological examination of lung tissue with (A) hematoxylin and eosin (H\&E) stain (magnification x200), (B) periodic acid-Schiff (PAS) (magnification x200), and (C) Masson's trichrome stain (magnification x200). Phosphate-buffered saline (PBS)/PBS; normal control mice treated with PBS only; OVA/PBS; OVA-sensitized/challenged mice treated orally with PBS; OVA/montelukast (Mon); OVA-sensitized/challenged mice treated orally with montelukast (30 mg/kg); OVA/BPTS-50; OVA-sensitized/challenged mice treated orally with BPTS (50 mg/kg); OVA/BPTS-100; OVA-sensitized/challenged mice treated orally with BPTS $(100 \mathrm{mg} / \mathrm{kg}){ }^{* * *}$ Significantly different from PBS/PBS, P<0.01; \#,\#\# significantly different from OVA/PBS, $\mathrm{P}<0.05$ and $<0.01$, respectively.

of total IgE and OVA-specific IgE in BALF and plasma were much higher in the OVA/PBS mice than in the PBS/PBS mice (Table II). The BPTS-treated mice had significantly lower levels of total IgE and OVA-specific IgE in BALF and plasma compared with the OVA/PBS mice.

BPTS decreases inflammatory infiltration, mucus hypersecretion and peribronchial collagen deposition. Histological analysis of the lung sections of the OVA/PBS mice showed dense inflammatory cell infiltration into the perivascular and peribronchial regions, as well as increased mucus secretion (Fig. 3A and B). The OVA/PBS mice had significantly greater subepithelial and interstitial fibrosis compared with the PBS/PBS mice, as assessed by Masson's trichrome staining
(Fig. 3C). Collagen levels in lung tissue, as assessed by ELISA, were significantly higher in the OVA/PBS mice (Fig. 3D). The OVA-challenged mice treated with BPTS presented significantly lower levels of inflammatory cell infiltration, mucus hypersecretion and peribronchial fibrosis (including collagen levels) compared with the OVA/PBS mice.

BPTS decreases TGF- $\beta 1$ and Smad3 expression levels in lung tissue. To identify the possible mechanisms underlying the inhibitory effects of BPTS on pulmonary fibrosis, TGF- $\beta 1$ and Smad3 expression levels were examined in relation to the fibrotic lung response of OVA/PBS mice. Western blot analysis revealed that TGF- $\beta 1$ (Fig. 4A) and Smad3 (Fig. 5A and B) protein expression levels were significantly higher in the lung 

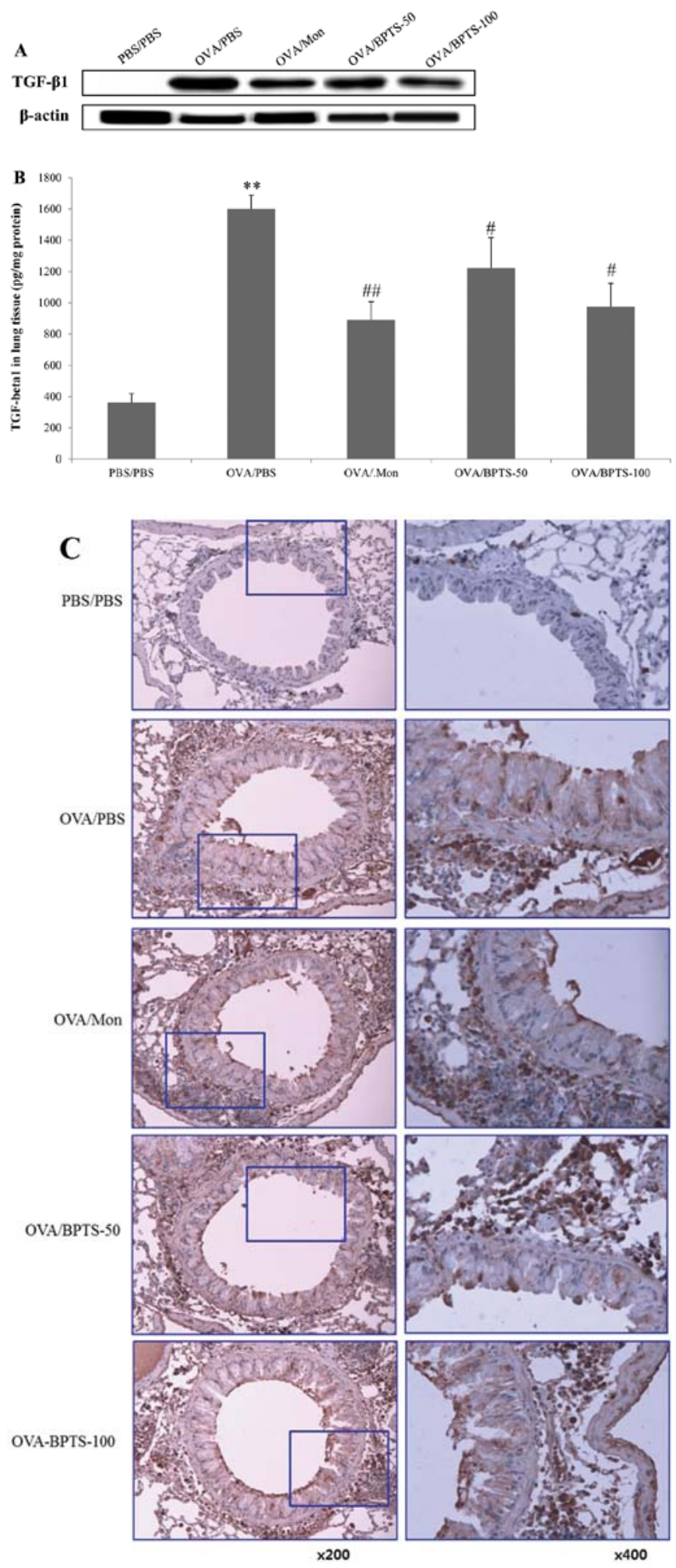

Figure 4. Bangpungtongseong-san water extract (BPTS) reduces the expression of TGF- $\beta 1$ levels in lung tissues $48 \mathrm{~h}$ after the final ovalbumin (OVA) challenge. (A) TGF- $\beta 1$ protein expression levels in immunoblotting, (B) TGF- $\beta 1$ expression levels in bronchoalveolar lavage fluid (BALF) measured by ELISA, (C) TGF- $\beta 1$ immunoreactivity in lung tissue. Right panels show a higher magnification image of the area indicated by the square box in the left panels. Phosphate-buffered saline (PBS)/PBS; normal control mice treated with PBS only; OVA/PBS; OVA-sensitized/challenged mice treated orally with PBS; OVA/montelukast (Mon); OVA-sensitized/challenged mice treated orally with montelukast (30 mg/kg); OVA/BPTS-50; OVA-sensitized/challenged mice treated orally with BPTS $(50 \mathrm{mg} / \mathrm{kg})$; OVA/BPTS-100; OVA-sensitized/ challenged mice treated orally with BPTS $(100 \mathrm{mg} / \mathrm{kg})$. Values are expressed as the means \pm SEM ( $n=7 /$ group). ${ }^{* *}$ Significantly different from PBS/PBS, $\mathrm{P}<0.01$;

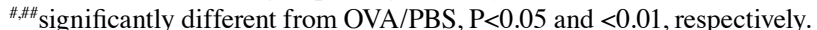
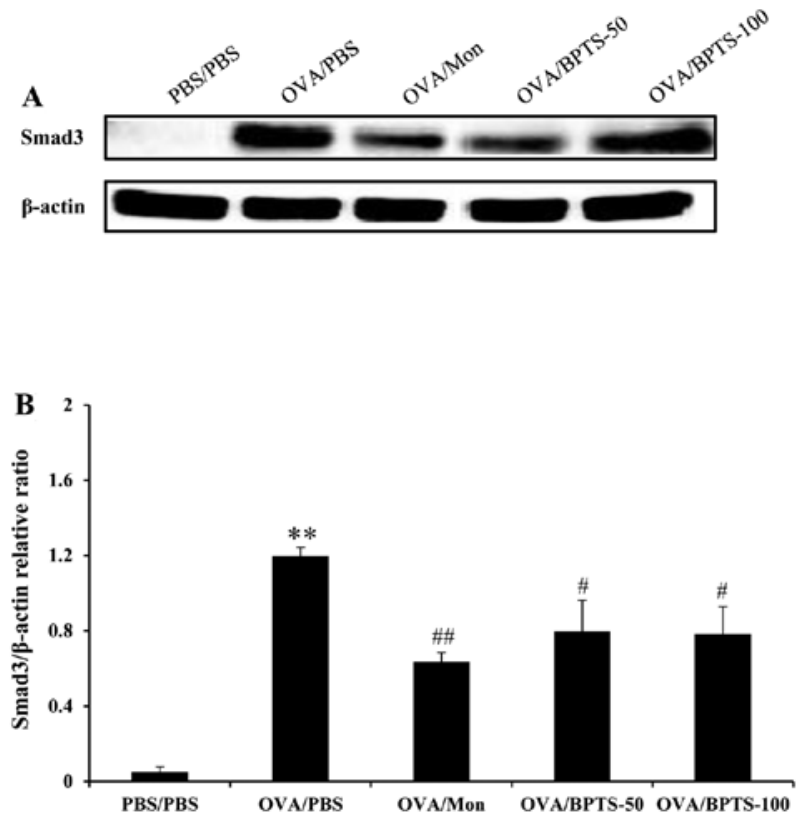

Figure 5. Bangpungtongseong-san water extract (BPTS) reduces Smad3 expression levels in lung tissues $48 \mathrm{~h}$ after the final ovalbumin (OVA) challenge. (A) Smad3 protein expression as assessed by immunoblotting. (B) Relative units are expressed as the relative ratio of $\operatorname{Smad} 3$ to $\beta$-actin. Phosphate-buffered saline (PBS)/PBS; normal control mice treated with PBS only; OVA/PBS; OVA-sensitized/challenged mice treated orally with PBS; OVA/montelukast (Mon); OVA-sensitized/challenged mice treated orally with montelukast (30 mg/kg); OVA/BPTS-50; OVA-sensitized/challenged mice treated orally with BPTS $(50 \mathrm{mg} / \mathrm{kg})$; OVA/BPTS-100; OVA-sensitized/challenged mice treated orally with BPTS $(100 \mathrm{mg} / \mathrm{kg})$. Values are expressed as the means \pm SEM ( $n=7 /$ group). ${ }^{* *}$ Significantly different from $P B S / P B S, P<0.01$; \#,\#\#significantly different from OVA/PBS, $\mathrm{P}<0.05$ and $<0.01$, respectively.

tissues of OVA/PBS mice compared with those of PBS/PBS mice, after they had undergone the final OVA challenge. BPTS treatment led to a significant attenuation of the increased TGF- $\beta 1$ expression levels compared with the OVA-challenged mice treated with PBS (Fig. 4A). These results were consistent with those obtained by ELISA analysis of the lung tissue (Fig. 4B) and immunohistochemistry (Fig. 4C).

BPTS decreases the expression levels of VEGF in BALF and lung tissue. As shown in Fig. 6A, the VEGF expression levels in the lung tissue were significantly higher in the OVA/PBS mice compared with the PBS/PBS mice. The level of VEGF was significantly lower in the BPTS-treated mice compared with the OVA/PBS mice. Consistent with these results, the immunoblot analysis results (Fig. 6B and C) and immunohistochemistry (Fig. 6D) of the lung sections revealed that VEGF was more abundant in the epithelial cells and inflammatory cells of the OVA/PBS mice compared with those of the PBS/ PBS mice. The BPTS-treated mice showed a reduced VEGF expression compared with the untreated OVA-challenged mice.

BPTS decreases the expression levels of VCAM-1 and ICAM-1 in lung tissue. To determine whether BPTS protects against asthmatic effects through VEGF, we investigated the expression of adhesion molecules related to inflammatory cell infiltration in the lungs. As shown in Fig. 7, the expression levels of ICAM-1 and VCAM-1 in the lung tissue were signifi- 

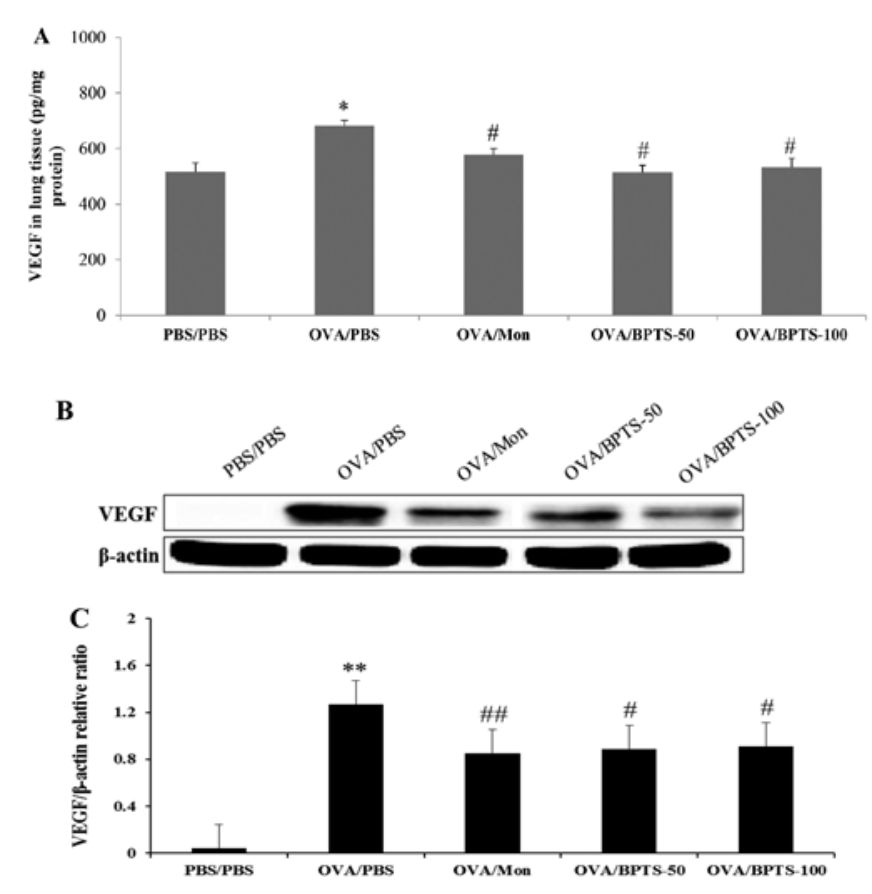

Figure 6. Bangpungtongseong-san water extract (BPTS) reduces the expression of VEGF in lung tissues $48 \mathrm{~h}$ after the final ovalbumin (OVA) challenge. (A) VEGF levels in BALF measured by ELISA. (B) Protein expression as assessed byimmunoblotting. (C) Relative units are expressed as the relative ratio of VEGF to $\beta$-actin. (D) VEGF immunoreactivity in lung tissue. Right panels show a higher magnification image of the area indicated by the square box in the left panels. Phosphate-buffered saline (PBS)/PBS; normal control mice treated with PBS only; OVA/PBS; OVA-sensitized/challenged mice given oral PBS; OVA/montelukast (Mon); OVA-sensitized/challenged mice given oral montelukast (30 mg/kg); OVA/BPTS-50; OVA-sensitized/challenged mice given oral BPTS $(50 \mathrm{mg} / \mathrm{kg})$; OVA/BPTS-100; OVA-sensitized/ challenged mice given oral BPTS $(100 \mathrm{mg} / \mathrm{kg})$. Values are expressed as the means \pm SEM ( $=7 /$ group). ${ }^{* *}$ Significantly different from PBS/PBS, $\mathrm{P}<0.01$;

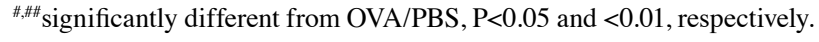

cantly higher in the OVA/PBS mice compared with the PBS/ PBS mice. BPTS treatment reduced the expression of VCAM-1 (Fig. 7A and B) and ICAM-1 (Fig. 7C and D) compared with control the OVA/PBS mice.

\section{Discussion}

In the present study, we evaluated the effects of BPTS in a murine model of experimental chronic OVA-induced asthma. We measured the levels of Th2 cytokines, chemokines and IgE, and assessed the histopathological changes following treatment with BPTS in OVA-challenged mice. To determine whether BPTS acts through an anti-fibrotic mechanism, immunoblot analysis and immunohistochemistry were performed to measure the abundance of adhesion molecules (VCAM- 1 and ICAM-1), VEGF, TGF- $\beta 1$ and Smad3 proteins in lung tissue. Chronic exposure of the OVA-challenged mice to BPTS reduced airway inflammatory cell infiltration, as well as the levels of cytokines, chemokines and IgE, collagen deposition, and the expression levels of VEGF, adhesion molecules, TGF- $\beta 1$ and Smad3.

Chronic asthma is characterized by airway inflammation, remodeling of the airways and hyperresponsiveness to environmental stimuli. Chronic asthma is accompanied by

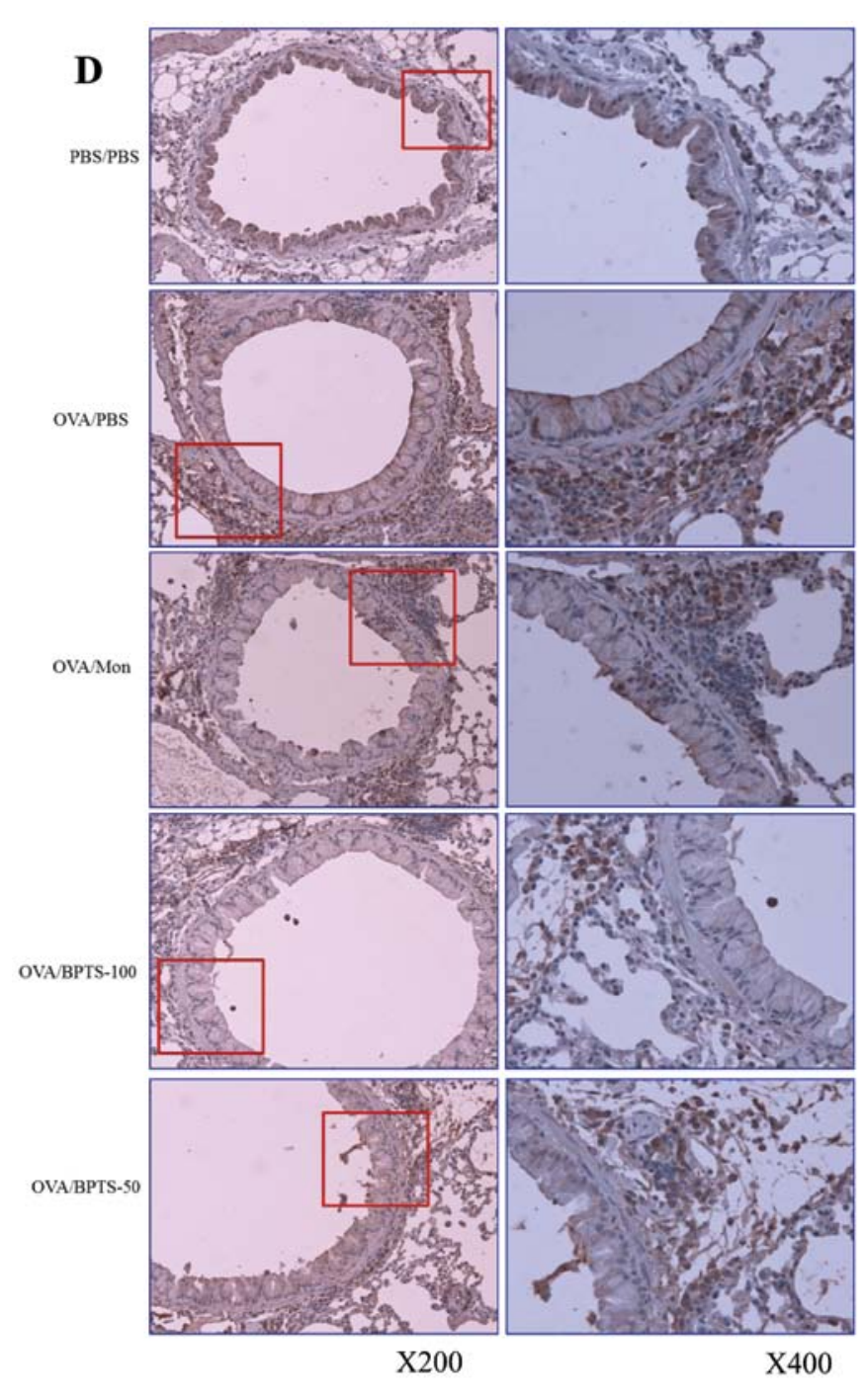

thickening of the bronchial walls, epithelial damage, subepithelial fibrosis, increased deposition of extracellular matrix proteins, and increased activity of various cytokines and growth factors, all of which contribute to airway remodeling. Airway inflammation is a hallmark characteristic of chronic asthma and is closely associated with Th2 cell activation and the release of cytokines, including IL-4, IL-5 and IL-13, which in turn accelerate the airway inflammatory cell infiltration (23). At elevated levels, Th2 cytokines can stimulate the production of growth factors, including VEGF and TGF- $\beta 1$, thereby resulting in pulmonary fibrosis $(24,25)$.

In our study, the administration of BPTS was found to reduce airway inflammatory cell infiltration and attenuate mucus hypersecretion by decreasing the $\mathrm{Th} 2$ cytokine and $\operatorname{IgE}$ expression levels. Histopathological analysis supported these findings, showing that BPTS attenuated both inflammatory cell infiltration in the peribronchial and perivascular regions, as well as the associated mucus production. According to these results, the administration of BPTS may suppress an ongoing Th2 2 response in chronic asthma.

In the current study, OVA-challenged mice displayed the pathophysiological features of chronic asthma, including airway inflammation, bronchial wall remodeling, elevated levels of Th2 cytokines, increased collagen concentration and 

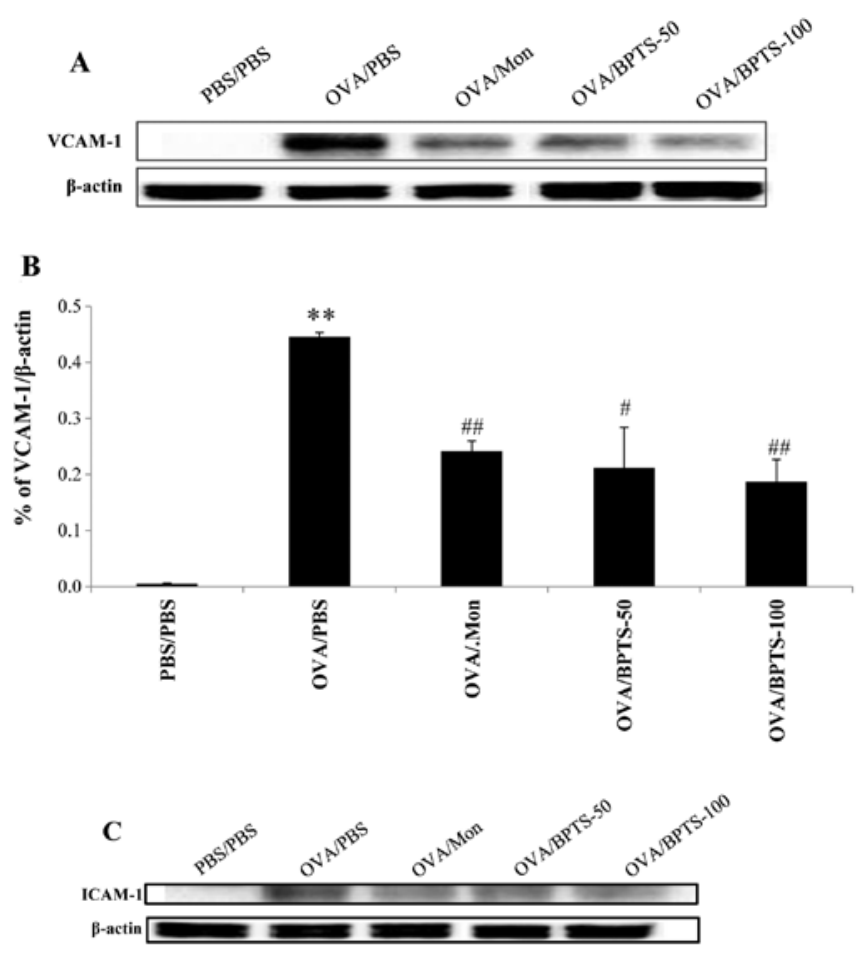

D

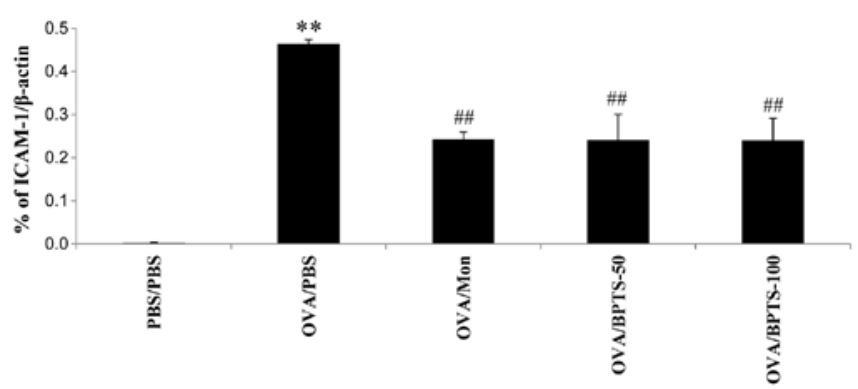

Figure 7. Bangpungtongseong-san water extract (BPTS) reduces vascular cell adhesion molecule 1 (VCAM-1) and intercellular adhesion molecule 1 (ICAM-1) expression in lung tissues $48 \mathrm{~h}$ after the final ovalbumin (OVA) challenge. (A) VCAM-1 protein expression as assessed by immunoblotting. (B) Relative units are expressed as the relative ratio of VCAM-1 to $\beta$-actin. (C) ICAM-1 protein expression as assessed by immunoblotting.(D) Relative units are expressed as the relative ratio of ICAM- 1 to $\beta$-actin. Phosphatebuffered saline (PBS)/PBS; normal control mice treated with PBS only; OVA/PBS; OVA-sensitized/challenged mice given oral PBS; OVA/montelukast (Mon); OVA-sensitized/challenged mice given oral montelukast (30 mg/kg); OVA/BPTS-50; OVA-sensitized/challenged mice given oral BPTS (50 mg/kg); OVA/BPTS-100; OVA-sensitized/challenged mice given oral BPTS $(100 \mathrm{mg} / \mathrm{kg})$. Values are expressed as means \pm SEM ( $\mathrm{n}=7 /$ group). ${ }^{* *}$ Significantly different from PBS/PBS, $\mathrm{P}<0.01$; \#,\#\# significantly different from OVA/PBS, $\mathrm{P}<0.05$ and $<0.01$, respectively

increased levels of VEGF and TGF- $\beta 1$. BPTS reduced the concentration of both IL-4 and IL-13 in BALF. These Th2 cytokines stimulate bronchial epithelia to secrete TGF- $\beta 1$, by passing the need for inflammatory cells (26). The Th2 cytokine level reduction is closely linked to the expression levels of growth factors, such as VEGF and TGF- $\beta 1$, which are considered to be important in the pathophysiological process of chronic asthma. Bronchial wall remodeling, a fundamental factor in the development of asthma, results from abnormal differentiation patterns of bronchial cells, in particular fibro- blasts $(27,28)$. Cellular responses induce IgE switching, which leads to mucus hypersecretion from goblet cells (22). As shown by the results of the current study, the administration of BPTS reduced airway inflammatory cell infiltration and attenuated mucus hypersecretion by decreasing Th 2 cytokine and $\operatorname{IgE}$ expression levels. Inflammatory cell infiltration and mucus hypersecretion are regulated by the elevated production levels of proinflammatory cytokines, in particular TGF- $\beta$, in regions of epithelial damage, as reflected in BALF from asthmatics (29). Our finding that BPTS reduced VEGF, TGF- $\beta 1$ and Smad3 expression levels in the lung tissue are consistent with their roles in chronic asthma.

TGF- $\beta 1$ is an important fibrogenic and immunomodulatory factor that is considered to play a pivotal role in the pathogenesis of airway remodeling (30). Cytokine TGF- $\beta 1$ concentration is elevated in asthma, and this increase is thought to be involved in the airway pathology associated with asthma (31). The release of TGF- $\beta 1$ is regulated by eosinophils and is stimulated by environmental factors, such as allergens and cigarette smoke (32). Therefore, inhibiting the production of TGF- $\beta 1$ or blocking its bioactivity in order to suppress airway collagen formation is considered a potential treatment strategy for preventing airway remodeling $(12,33)$.

In asthma, the increased vascularization of the airway walls correlates with an increased VEGF expression in the airways (34). Lee et al (24) reported direct evidence for the involvement of VEGF in lung tissue remodeling. VEGF is a multifunctional cytokine required for endothelial cell survival that has potent actions on vascular endothelial cells, including increased vascular permeability, the induction of endothelial cell mitogenesis and increased cell migration $(35,36)$.

Consistent with previous studies, the present study showed that OVA challenge increased TGF- $\beta 1$ and VEGF expression levels and decreased the airway migration of eosinophils. These data suggest that TGF- $\beta 1$ inhibition is regulated by the suppression of eosinophil migration. Thus, treatment with BPTS may prevent pulmonary fibrosis in lung tissue by inhibiting collagen deposition. These observations are consistent with our findings that VEGF, TGF- $\beta 1$ and Smad3 protein expression levels were upregulated, and pulmonary fibrosis was increased in OVA-challenged mice. By contrast, the administration of BPTS significantly blocked the pulmonary fibrosis induced in OVA-challenged mice by reducing VEGF, TGF- $\beta 1$, and Smad 3 expression levels in lung tissue. These results suggest that BPTS attenuates pulmonary fibrosis induced by repeated OVA challenge, at least partly by inhibiting VEGF and TGF- $\beta 1-$ Smad 3 signaling.

In conclusion, the administration of BPTS reduces airway inflammation and collagen deposition by reducing Th2 cytokine release and inhibiting the expression of VEGF, TGF- $\beta 1$ and Smad3 in lung tissue from OVA-challenged mice. These data suggest that BPTS effectively protects against chronic asthma symptoms, such as inflammatory cell infiltration, mucus-secreting goblet cell hyperplasia and collagen deposition in the airways, which are induced by repeated OVA challenge.

\section{Acknowledgements}

This research was part of a project (The Evidence Based Medicine for Herbal Formula) funded by the Basic Herbal 
Medicine Research Group in the Korea Institute of Oriental Medicine.

\section{References}

1. Busse WW and Lemanske RF Jr: Asthma. N Engl J Med 344: 350-362, 2001.

2. Rincon M and Irvin CG: Role of IL-6 in asthma and other inflammatory pulmonary diseases. Int J Biol Sci 8: 1281-1290, 2012.

3. Finkelman FD, Holmes J, Urban JF Jr, Paul WE and Katona IM: T help requirements for the generation of an in vivo IgE response: a late acting form of T cell help other than IL-4 is required for IgE but not for IgG1 production. J Immunol 142: 403-408, 1989.

4. Adachi T, Motojima S, Hirata A, Fukuda T and Makino S: Eosinophil viability-enhancing activity in sputum from patients with bronchial asthma, Contributions of interleukin-5 and granulocyte/macrophage colony-stimulating factor. Am J Respir Crit Care Med 151: 618-623, 1995.

5. Sur S, Kita H, Gleich GJ, Chenier TC and Hunt LW: Eosinophil recruitment is associated with IL-5, but not with RANTES, twenty-four hours after allergen challenge. J Allergy Clin Immunol 97: 1272-1278, 1996.

6. Oettgen HC and Geha RS: IgE in asthma and atopy: cellular and molecular connections. J Clin Invest 104: 829-835, 1999.

7. Nelms K, Keegan AD, Zamorano J, Ryan JJ and Paul WE: The IL-4 receptor: signaling mechanisms and biologic functions. Annu Rev Immunol 17: 701-738, 1999.

8. Zurita-Salinas CS, Palacios-Boix A, Yáñez A, González F and Alcocer-Varela J: Contamination with Mycoplasma spp. induces interleukin-13 expression by human skin fibroblasts in culture. FEMS Immunol Med Microbiol 15: 123-128, 1996.

9. Botero JE, Contreras A and Parra B: Profiling of inflammatory cytokines produced by gingival fibroblasts after human cytomegalovirus infection. Oral Microbiol Immunol 23: 291-298, 2008.

10. Wilson MS and Wynn TA: Pulmonary fibrosis: pathogenesis, etiology and regulation. Mucosal Immunol 2: 103-121, 2009.

11. Mauviel A: Transforming growth factor beta: a key mediator of fibrosis. Meth Mol Med 117: 69-80, 2005.

12. Minshall EM, Leung DY, Martin RJ, Song YL, Cameron L, Ernst P and Hamid Q: Eosinophil-associated TGF-betal mRNA expression and airways fibrosis in bronchial asthma. Am J Respir Cell Mol Biol 17: 326-333, 1997.

13. Vasquez-Pinto LM, Nantel F, Sirois P and Jancar S: Bradykinin $\mathrm{B}$ (1) receptor antagonist $\mathrm{R} 954$ inhibits eosinophil activation/ proliferation/migration and increases TGF-beta and VEGF in a murine model of asthma. Neuropeptides 44: 107-113, 2010.

14. Lee KS, Park SJ, Kim SR, Min KH, Lee KY, Choe YH, Hong SH, Lee YR, Kim JS, Hong SJ and Lee YC: Inhibition of VEGF blocks TGF- $\beta 1$ production through a PI3K/Akt signaling pathway. Eur Respir J 31: 523-531, 2008.

15. Foster CA: VCAM-1/ $\alpha$ 4-integrin adhesion pathway: therapeutic target for allergic inflammatory disorders. J Allergy Clin Immunol 98: S270-S277, 1996.

16. Zhu X, Subbaraman R, Sano H, Jacobs B, Sano A, Boetticher E, Muñoz NM and Leff AR: A surrogate method for assessment of $\beta 2$-integrin-dependent adhesion of human eosinophils to ICAM-1. J Immunol Methods 240: 157-164, 2000.

17. Shimada T, Kudo T, Akase T and Aburada M: Preventive effects of Bofutsushosan on obesity and various metabolic disorders. Biol Pharm Bull 31: 1362-1367, 2008.

18. Ohno K, Chung H J, Maruyama I and Tani T: Bofutsushosan, a traditional Chinese formulation, prevents intimal thickening and vascular smooth muscle cell proliferation induced by balloon endothelial denudation in rats. Biol Pharm Bull 28: 2162-2165, 2005.
19. Morimoto Y, Sakata M, Ohno A, Maegawa T and Tajima S: Effects of Byakko-ka-ninjin-to, Bofu-tsusho-san and Gorei-san on blood glucose level, water intake and urine volume in KKAy mice. Yakugaku Zasshi 122: 163-168, 2002 (In Japanese).

20. Kim HJ, Yoon KM, Im EY, Byun JS, Kim DJ and Kwak MA: Three case report of Bangpungtongsung-san effect on improvement of hypertension patients. KJOPP 23: 740-743, 2009.

21. Kim HJ, Park OS, Kim KS, Cha JH and Kim YB: The effect of Bangpungtongsung-San on model of allergic rhinitis. JKOOD 19: 21-30, 2006.

22. Lee MY, Shin IS, Lim HS, Seo CS, Ha H and Shin HK: Kochia scoparia fruit attenuates allergic airway inflammation in ovalbumin (OVA)-induced murine asthma model. Inhal Toxicol 23: 938-946, 2011.

23. Yuk JE, Lee MY, Kwon OK, Cai XF, Jang HY, Oh SR, Lee HK and Ahn KS: Effects of astilbic acid on airway hyperresponsiveness and inflammation in a mouse model of allergic asthma. Int Immunopharmacol 11: 266-273, 2011.

24. Lee G, Link H, Baluk P, Homer RJ, Chapoval S, Bhandari V, Kang MJ, Cohn L, Kim YK, McDonald DM and Elias JA: Vascular endothelial growth factor (VEGF) induces remodeling and enhances TH2-mediated sensitization and inflammation in the lung. Nat Med 10: 1095-1103, 2004.

25. Chaudhary NI, Roth GJ, Hilberg F, Müller-Quernheim J, Prasse A, Zissel G, Schnapp A and Park JE: Inhibition of PDGF, VEGF and FGF signaling attenuates fibrosis. Eur Respir J 29: 976-985, 2007.

26. Richter A, Puddicombe SM, Lordan JL, Bucchieri F, Wilson SJ, Djukanovic R, Dent G, Holgate ST and Davies DE: The contribution of interleukin (IL)-4 and IL-13 to the epithelialmesenchymal trophic unit in asthma. Am J Respir Cell Mol Biol 25: 385-391, 2001.

27. Sumi Y and Hamid Q: Airway remodeling in asthma. Allergol Int 56: 341-348, 2007.

28. Westergren-Thorsson G, Larsen K, Nihlberg K, Andersson-Sjöland A, Hallgren O, Marko-Varga $G$ and Bjermer L: Pathological airway remodelling in inflammation. Clin Respir J 4: 1-8, 2010.

29. Howell JE and McAnulty RJ: TGF- $\beta$ : its role in asthma and therapeutic potential. Current Drug Targets 7: 547-565, 2006.

30. Halwani R, Al-Muhsen S, Al-Jahdali H and Hamid Q: Role of transforming growth factor- $\beta$ in airway remodeling in asthma. Am J Respir Cell Mol Biol 44: 127-133, 2011.

31. Salib RJ and Howarth PH: Transforming growth factor- $\beta$ in allergic inflammatory disease of the upper airways: friend or foe? Clin Exp Allergy 39: 1128-1135, 2009.

32. Baek KJ, Cho JY, Rosenthal P, Alexander LE, Nizet V and Broide DH: Hypoxia potentiates allergen induction of HIF- $1 \alpha$, chemokines, airway inflammation, TGF- $\beta 1$, and airway remodeling in a mouse model. Clin Immunol 147: 27-37, 2013.

33. Yamaguchi M, Niimi A, Matsumoto H, Ueda T, Takemura M, Matsuoka H, Jinnai M, Otsukda K, Oguma T, Takeda T, Ito I, Chin K and Mishima M: Sputum levels of transforming growth factor- $\beta 1$ in asthma: relation to clinical and computed tomography findings. J Investig Allergol Clin Immunol 18: 202-206, 2008.

34. Hoshino M, Takahashi M and Aoike N: Expression of vascular endothelial growth factor, basic fibroblast growth factor, and angiogenin immunoreactivity in asthmatic airways and its relationship to angiogenesis. J Allergy Clin Immunol 107: 295-301, 2001.

35. Chavakis E and Dimmeler S: Regulation of endothelial cell survival and apoptosis during angiogenesis. Arterioscler Thromb Vasc Biol 22: 887-893, 2002.

36. Ferrara N, Gerber HP and Le Couter J: The biology of VEGF and its receptors. Nat Med 9: 669-676, 2003. 